\title{
Potential applications of next generation sequencing to the genomics of Posidonia oceanica
}

\author{
Andrea Cavallini*, Lucia Natali and Tommaso Giordani \\ Department of Agricultural, Food, and Environmental Sciences, University of Pisa, Pisa, Italy \\ *Correspondence: acavalli@agr.unipi.it \\ Edited by: \\ Silvia Mazzuca, Università della Calabria, Italy \\ Reviewed by: \\ Silvia Mazzuca, Università della Calabria, Italy
}

\section{MOLECULAR STUDIES ON Posidonia oceanica ARE STILL LIMITED}

Despite of its paramount ecological importance in shallow shoreline habitats in the Mediterranean sea, basic knowledge of Posidonia oceanica genome is still limited, with regard to both functional and structural genomics.

Concerning protein-coding sequences, a survey of NCBI nucleotide database resulted in finding only 103 putative genes (on November 5, 2012). Of these, many are putative metallothionein (MT)- or aquaporin (AQP)-encoding genes.

These sequences have been extensively studied, since involved in the adaptation of Posidonia to endangered environments, at both physiological and genetic levels. Sequences encoding putative type2 MTs belong to a multigene family with at least two subgroups (Giordani et al., 2000; Cozza et al., 2006). Northern hybridizations indicated that MT transcripts accumulation is constitutive and seasonally regulated. However, MT transcripts accumulated after rhyzome harvesting and after 15 days of cultivation in an aquarium. As for animal MTs, transcripts accumulation was observed also after exposure to trace metals such as copper and cadmium. The two MT subgroups showed differences in their histological expression, i.e., in proliferative tissues or in lignified or suberized cells.

Concerning AQP gene family, two genes encoding AQPs of the plasmalemma and the tonoplast were isolated (Maestrini et al., 2004). Both genes were constitutively expressed in the leaves, with higher levels of transcripts in young than in differentiated leaf tissues. Variations of salt concentration in aquarium determined different AQPs transcript accumulation, suggesting that $\mathrm{AQPs}$ are involved in osmotic balance maintenance in seagrasses (Maestrini et al., 2004; Serra et al., 2011).

When surveying the Posidonia repetitive DNA, i.e., the intergenic, putatively non-coding portion of the genome, one can realize that this genome component is even less studied than coding portion. The 2C-genome size of Posidonia was established in 5-6 pg DNA (Cavallini et al., 1995; Koce et al., 2003), posing this species in the middle range of monocots, being genome much larger than in other seagrasses as Cymodocea nodosa (1.1 pg), Zostera nolti (1.5 pg), and $Z$. marina $(1.2 \mathrm{pg}$ ) (Koce et al., 2003). Such differences are largely independent on chromosome number and hence on polyploidization: $P$. oceanica has $2 n=20$ and Zostera species have $2 n=12$ (Koce et al., 2003). P. oceanica genome has been characterized biochemically (Cavallini et al., 1995; Maestrini et al., 2002). At molecular level, only a few repetitive DNA sequences are found in the NCBI nucleotide database.

\section{PRESENT STATUS OF P. oceanica GENOMICS}

During last years, the research for coding sequences in $P$. oceanica has proceeded through the production of both differential and EST libraries for obtaining sequences to be used in analyses of environmental adaptation of Posidonia meadows, especially associated with different light and temperature regimes and other potential stress-responsive gene networks. A SSH-cDNA library from a continuous meadow at two different depths $(-5$ and $-25 \mathrm{~m})$ produced 486 tentative unigenes, of which only 28 were common to both shallow and deep libraries (Dattolo et al., 2009). All genes were grouped in functional classes showing the highest differences between the two depths in primary metabolism, photosynthesis and stress defence genes.

Another recent study deals with the methylation status of $P$. oceanica chromosomal DNA, analysed by applying a Methylation-Sensitive Amplification Polymorphism technique, a modification of well-known AFLP procedure (Greco et al., 2012). This analysis allowed to identify a few loci that are differently methylated between Cd-treated and control plants.

In other experiments, a massive sequencing effort produced the first seagrass sequence database, Dr. Zompo, based on 14,597 ESTs obtained from Z. marina $(9,412 \mathrm{ESTs})$ and P. oceanica $(5,185)$ (Wissler et al., 2009). Dr. Zompo constitutes a valuable resource for the seagrass community, and is expected to grow in the near future, particularly with the recent initiation of the Zostera genome sequencing project.

Zostera marina sequencing initiative has started in 2010 under the guide of Dr. J. Olsen (University of Groningen) and it will greatly increase basic knowledge of seagrasses, allowing a deeper knowledge of seagrass biology and favoring studies in which analyses of genetic variability will be associated to the functional significance of differences.

Though sequencing of $Z$. marina genome will produce important consequences for all seagrasses, it is to be recalled that, from the genetic side, $P$. oceanica genome is much larger than that of Z. marina (around 5-fold). Major differences in genome size are generally related to polyploidization and/or to the amplification of non-coding, repetitive DNA, as for example centromeric and telomeric tandem repeats and, especially, 
transposable elements. Considering the different $2 \mathrm{n}$-chromosome number of the two seagrass species (20 vs. 12), the large difference in genome size should be especially related to differences in repetitive DNA, though a cryptopolyploidy event cannot be excluded.

Repetitive DNA has long been considered as "selfish," providing no adaptive benefit to the host genome. In the last years, repetitive DNA has shown to be essential for genome function. For example, transposons have a role in genome restructuring (Kazazian, 2000). Gene fragments rearranged by transposons could be the primary mechanism for generating new genes (Morgante et al., 2005). Other transposons effects on genome structure range from providing promoter and enhancer activity to modulating transcript elongation, contributing to pericentromeric and intercalary heterochromatin, supplying chromatin boundary signals for heterochromatin domains and hence playing a major architectonic role in higher order physical structuring of the nucleus (Von-Sternberg and Shapiro, 2005).

The influence of transposons on gene activity appears to be of special importance. Usually, phenotypic variation is conceived in terms of altered gene products produced by mutations in protein-coding sequences. However, other mechanisms generating variability do occur. For example, the organization of proteins can change without coding sequence modifications through changes in RNA splicing patterns via the integration of retroelements into introns (Nekrutenko and Li, 2001).

Transposon movements can change the regulatory formatting of conserved coding sequences. Such changes are even more important than those previously described, resulting in novel developmental patterns and new traits using the same assemblage of proteins and RNAs. In model organisms as the mouse and Arabidopsis, genetic studies on development have shown that retroelements play a role in the epigenetic settings of the genome, both globally, regulating chromatin organization in the nucleus (Van-Driel et al., 2003), and locally, as control elements of the expression of genes (Song et al., 2004). It appears that when the repetitive component of the genome changes, the epigenetic settings are modified, resulting in changes of phenotypic traits (Chong and Whitelaw, 2004).

\section{NGS TECHNIQUES AND PERSPECTIVES FOR Posidonia GENOMICS}

Large size and high content of repetitive DNA elements are the major obstacles to genome sequencing: it is preferable to sequence model species with small genomes as Zostera and a concomitant similar initiative for Posidonia is not realistic at present.

Non-model species can be, however, conveniently explored using novel methods that imply next generation sequencing and bioinformatic analyses. Next generation sequencing apparently represents a step change and a starting point for genetical and biological research in the Twenty-first century, producing genomic sequences in parallel, at ever increasing speed and decreasing costs. So several Gigabases of data can be sequenced in a few weeks for a fraction of the costs of Sanger sequencing (Ansorge, 2009). NGS technologies offer novel, rapid ways for large, genome-wide characterization and profiling of DNA, mRNAs, small-RNAs, transcription factor regions, chromatin structure, and DNA methylation patterns.

For functional genomics studies, the next-generation technology allows the analysis of RNA transcripts by relatively short sequence tags, up to $150 \mathrm{nt}$ for Illumina and 400-500 nt for 454, directly from each cDNA in the sample. Owing to the huge number of samples analysed simultaneously, sequence-based techniques can detect low abundance RNAs, small RNAs, or the presence of rare cells contained in the sample. Another advantage of this approach is that it does not require prior knowledge of the genome sequence.

Applications include precise quantification of RNA transcripts, measured through their sequence, without the probe hybridization employed in DNA chip techniques; identification and analysis of DNA regions that interact with regulatory proteins in functional regulation of gene expression; recovery and analysis of all components of a gene family.

Limitations of this technology are short read lengths, non-uniform confidence in base calling in sequence reads, particularly deteriorating $3^{\prime}$-sequence quality and generally lower reading accuracy in homopolar stretches of identical bases. Another limiting step is related to the huge amount of unorganized sequence data generated by these systems, that require the development of software and efficient computer algorithms.

\section{NGS TECHNIQUES FOR STUDYING Posidonia STRUCTURAL GENOMICS}

The contribution of repetitive DNA to genome structure and function has been studied in completely sequenced genomes. However, many questions remain about the distribution of repetitive sequences and the overall genome organization in plants with medium-large genomes, in which repetitive DNA is most abundant and presumably most important. Monocotyledons other than Graminaceae have in general been given little attention, and knowledge of the repetitive DNA in the genome evolution in seagrasses is totally lacking.

NGS technology can be conveniently applied for the identification of sequences present in many copies per genome, by producing of large numbers of short and randomly placed sequences and assembling them according to their sequence.

Actually, the major computational task in de novo assembling of millions of reads is represented by reads that map to multiple locations (that is, multi-reads). A combination of strategies have to be used in genome assembly for resolving problems caused by repetitive DNA, for example sequencing strategies that use fragment libraries of varying sizes. However, in searching repetitive DNA families, a smaller coverage can allow reducing redundancy of reads and hence favouring their assembly into contigs, obtaining repeat identification and reconstruction. Using low genome coverage, most of the contigs that are obtained do not represent specific genomic loci; instead, they are probably composed of reads derived from multiple copies of repetitive elements, thus representing consensus sequences of genomic repeats (Novák et al., 2010). Even though the exact sequence of this consensus does not necessarily occur in the genome, this representation of repetitive elements has been shown to be sufficiently 
accurate to enable amplification of the whole length repetitive elements using PCR (Swaminathan et al., 2007).

We have mentioned only a few of the potentialities of NGS technology to structural genomics of seagrasses. Such studies are expected to greatly improve the understanding of molecular physiology and adaptation mechanisms of these plants and represent an excellent opportunity for rapidly overcoming the present lack of knowledge.

\section{REFERENCES}

Ansorge, W. J. (2009). Next-generation DNA sequencing techniques. N. Biotechnol. 25, 195-203.

Cavallini, A., Natali, L., Giordani, T., Polizzi, E., Balestri, E., Cinelli, F., et al. (1995). Cytophotometric and biochemical characterization of Posidonia oceanica L. (Potamogetonaceae) genome. Caryologia 48, 201-209.

Chong, S., and Whitelaw, E. (2004). Murine metastable alleles and transgenerational epigenetic inheritance. Cytogenet. Genome Res. 105, 311-315.

Cozza, R., Pangaro, T., Maestrini, P., Giordani, T., Natali, L., and Cavallini, A. (2006). Isolation of putative type 2-metallothionein encoding sequences and spatial expression pattern in the seagrass Posidonia oceanica. Aquat. Bot. 85, 317-323.

Dattolo, E., Innocenti, A. M., Mazzuca, S., Cavallini, A., Natali, L., and Procaccini, G. (2009). "Differential gene expression profiling under different light conditions in Posidonia oceanica (L.) Delile by SSH analysis," in Mediterranean Seagrass Workshop (Hvar), 19-20.

Giordani, T., Natali, L., Maserti, B. E., Taddei, S., and Cavallini, A. (2000). Characterization and expression of DNA sequences encoding putative type II metallothioneins in the seagrass Posidonia oceanica Delile. Plant Physiol. 123, 1571-1582.

Greco, M., Chiappetta, A., Bruno, L., and Bitonti, M. B. (2012). In Posidonia oceanica cadmium induces changes in DNA methylation and chromatin patterning. J. Exp. Bot. 63, 695-709.

Kazazian, H. H. (2000). L1 retrotransposons shape the mammalian genome. Science 289, 1152-1153.

Koce, J. D., Vilhar, B., Bohanec, B., and Dermastia, M. (2003). Genome size of Adriatic seagrasses. Aquat. Bot. 77, 17-25.

Maestrini, P., Giordani, T., Lunardi, A., Cavallini, A., and Natali, L. (2004). Isolation and expression of two aquaporin-encoding genes from the marine phanerogam Posidonia oceanica. Plant Cell Physiol. 45, 1838-1847.

Maestrini, P., Giordani, T., Polizzi, E., Natali, L., Maserti, B. E., Balestri, E., et al. (2002). Mercuryinduced DNA variations in the genome of Posidonia oceanica Delile. Caryologia 55, 47-53.

Morgante, M., Brunner, S., Pea, G., Fengler, K., Zuccolo, A., and Rafalski, A. (2005). Gene duplication and exon shuffling by helitron-like transposons generate intraspecies diversity in maize. Nat. Genet. 37, 997-1002.

Nekrutenko, A., and Li, W. H. (2001). Transposable elements are found in a large number of human protein coding regions. Trends Genet. 17, 619-625.

Novák, P., Neumann, P., and Macas, J. (2010). Graphbased clustering and characterization of repetitive sequences in next-generation sequencing data. BMC Bioinformatics 11:378. doi: 10.1186/14712105-11-378

Serra, I. A., Nicastro, S., Mazzuca, S., Natali, L., Cavallini, A., and Innocenti, A. M. (2011). Response to salt stress in seagrasses: PIP1;1 aquaporin antibody localization in Posidonia oceanica leaves. Aquat. Bot. Available online at: http://www.sciencedirect.com/science/article/pii/ S0304377011000878

Song, X., Sui, A., and Garen, A. (2004). Binding of mouse VL30 retrotransposon RNA to PSF protein induces genes repressed by PSF: effects on steroidogenesis and oncogenesis. Proc. Natl. Acad. Sci. U.S.A. 101, 621-626.

Swaminathan, K., Varala, K., and Hudson, M. E. (2007). Global repeat discovery and estimation of genomic copy number in a large, complex genome using a high-throughput 454 sequence survey. BMC Genomics 8:132. doi: 10.1186/1471-2164$8-132$

Van-Driel, R., Fransz, P. F., and Verschure, P. J. (2003). The eukaryotic genome: a system regulated at different hierarchical levels. J. Cell Sci. 116, 4067-4075.

Von-Sternberg, R., and Shapiro, J. A. (2005). How repeated retroelements format genome function. Cytogenet. Genome Res. 110, 108-116.

Wissler, L., Dattolo, E., Moore, A. D., Reusch, T. B. H., Olsen, J. L., Migliaccio, M., et al. (2009). Dr. Zompo: an online data repository for Zostera marina and Posidonia oceanica ESTs. Database 2009: bap009. doi: 10.1093/database/bap009

Received: 08 November 2012; accepted: 20 November 2012; published online: 05 December 2012

Citation: Cavallini A, Natali L and Giordani T (2012) Potential applications of next generation sequencing to the genomics of Posidonia oceanica. Front. Plant Sci. 3:273. doi: $10.3389 /$ fpls.2012.00273

This article was submitted to Frontiers in Plant Proteomics, a specialty of Frontiers in Plant Science. Copyright (c) 2012 Cavallini, Natali and Giordani. This is an open-access article distributed under the terms of the Creative Commons Attribution License, which permits use, distribution and reproduction in other forums, provided the original authors and source are credited and subject to any copyright notices concerning any third-party graphics etc. 